\title{
Non-Fragile Filtering Control Based on Output Feedback for Uncertain Linear Systems with Pole Constraints
}

\author{
Jian-Dong Yang \\ China Satellite Maritime Tracking and Control Department, \\ Jiangyin, 214431, China \\ E-mail: yjdjs250@126.com \\ Wei Huang \\ Management School, Shanghai University of Engineering \\ Science, Shanghai, 201620, China \\ E-mail: hw@sues.edu.cn
}

\author{
Xin-Yu Meng \\ College of Automation Engineering, Nanjing University of \\ Aeronautics and Astronautics, Nanjing, 210016, China \\ Northwest Institute of Nuclear Technology, Xi'an, 710024, \\ China \\ E-mail: mengxinyu@nuaa.edu.cn
}

\author{
Ming Ni, Fei Tao \\ Institute of Computer Science, National University of \\ Defense Technology, Changsha, 410073, China \\ China Satellite Maritime Tracking and Control Department, \\ Jiangyin, 214431, China \\ E-mail: niming_1989@126.com,347485192@qq.com
}

\begin{abstract}
For a class of linear systems with parametric uncertainties, we studied the design of the systems' robust and non-fragile filtering systems with closed-loop regional pole constraints, and proposed a design method for non-fragile filtering systems based on output feedback. The resulting closed-loop system is asymptotical stable, and $\mathbf{H}$-infinity disturbance attenuation satisfies a prescribed upper bound with respect to system uncertainty and filter gain perturbations. The closed-loop poles are assigned in an appointed region of the complex plane as well. Additive perturbations are considered. Solvability condition for the existence of filtering control system is derived in the form of a linear matrix inequality (LMI). Finally, this method is applied to an unmanned airship's longitudinal motion control, which also demonstrates the effectiveness of the proposed approach.
\end{abstract}

Keywords-output feedback; filtering control; non-fragile; pole constraints; LMI

\section{INTRODUCTION}

In some practical projects, such as unmanned aircraft flight-test, maybe the states can not be measured accurately or even lost because of state measuring sensors' failures or other reasons. These problems, as well as unable to control timely and effectively, demand setting an auxiliary program emergency switch to ensure continued flight or safe aircraft landing. To solve these problems, this paper proposes using an output feedback system to control a target system, and using the outputs of feedback system to track and estimate the outputs of controlled objects, which, as an emergency aid control scheme, replaces original control methods in order to promptly and effectively control unmanned aerial vehicles (UAV) or guarantee their safe return.

Theoretical researches on output feedback, filter (also involving robust tracking estimate of controlled output), and non-fragile control have obtained considerable fruits at home and abroad ${ }^{[1-5]}$. Aiming at the uncertainty of controlled system, literature [1] studied design issues for output feedback controllers with variance and pole constraints, which made systems stable through feedback control, as well as made the systems have certain dynamic response performance. However, controllers' uncertainty had not been taken into account in this literature, which would lead to some limitations in practical applications. Literature [2] studied the fuzzy output feedback control of uncertain systems, and obtained system stabilization conditions, but did not consider the uncertainty of controllers, either. Aiming at multiplicative filter parameters, literature [3] proposed Kalman filter design method for linear systems, but the results were complicated and the uncertainty of controlled systems was not taken into account. Literature [4] designed a linear continuous system $H_{\infty}$ filter, aiming at parameters change of additive filter, but the solving process was very complex. Literature [5] provided a design method of non-fragile filters for linear systems by using LMI method, but also ignored the uncertainty of controlled systems.

What is based on feedback filter control is yet rarely used in UAV flight control. In addition, in UAV flight control process, it also has certain requirements for the dynamic performance of the system. This paper proposes a design method for feedback filter control systems by using linear matrix inequality approaches, with the uncertainty of UAV models and filtering systems, and broader quadratic matrix inequality regions being taken into account.

\section{DESCRIPTION OF THE PROBLEM}

Considering the linear model of unmanned aerial vehicles described by the state-space: 


$$
\left\{\begin{array}{l}
\dot{x}(t)=(A+\Delta A) x(t)+(B+\Delta B) u(t)+D \omega(t) \\
y(t)=C_{1} x(t) \\
z(t)=C_{2} x(t)
\end{array}\right.
$$

Where $x \in R^{n}$ is a system state vector, $u \in R^{p}$ is a control input, $\omega \in R^{r}$ is an energy-bounded interference signal, $z \in R^{m}$ is a controllable output, $y(t) \in R^{q}$ is a measuring output, $A, B, C_{1}$ and $C_{2}$ are the known system matrixes.

Then construct the form of full-rank output feedback filter system:

$$
\left\{\begin{array}{l}
\dot{x}_{k}(t)=\left(A_{k}+\Delta A_{k}\right) x_{k}(t)+\left(B_{k}+\Delta B_{k}\right) y(t) \\
u(t)=C_{k 1} x_{k}(t) \\
z_{k}(t)=C_{k 2} x_{k}(t)
\end{array}\right.
$$

Where $x_{k} \in R^{n}$ is the state vector of the feedback control system, $z_{k}$ is the tracking of $z$, and $A_{k}, B_{k}, C_{k 1}$ and $C_{k 2}$ are the matrix parameters to be evaluated. Let the UAV model and the uncertain parameters, $\Delta A, \Delta B$ and $\Delta A_{k}, \Delta B_{k}$, of the filter system be norm-bounded and have the following perturbation form:

$$
\left[\begin{array}{cc}
\Delta A & \Delta B \\
\Delta A_{k} & \Delta B_{k}
\end{array}\right]=\left[\begin{array}{l}
E_{1} \\
E_{2}
\end{array}\right] H\left[\begin{array}{ll}
F_{1} & F_{2}
\end{array}\right]
$$

$E_{1}, E_{2}$ and $F_{1}, F_{2}$ are known as phalanxes to have appropriate dimensions, $H$ is an unknown real matrix which meets $H^{T}(t) H(t) \leq I$. The filtering error dynamic system is:

$$
\left\{\begin{array}{l}
\dot{\bar{X}}(t)=(\bar{A}+\Delta \bar{A}) \bar{X}(t)+\bar{D} \omega(t) \\
e(t)=z(t)-z_{k}(t)=\bar{C} \bar{X}(t)
\end{array}\right.
$$

Where

$$
\begin{gathered}
\bar{x}(t)=\left[\begin{array}{l}
x(t) \\
x_{k}(t)
\end{array}\right], \bar{A}=\left[\begin{array}{cc}
A & B C_{k 1} \\
B_{k} C_{1} & A_{k}
\end{array}\right], \bar{D}=\left[\begin{array}{c}
D \\
0
\end{array}\right], \\
\bar{C}=\left[\begin{array}{ll}
C_{2} & -C_{k 2}
\end{array}\right], \\
\overline{\Delta A}=\left[\begin{array}{cc}
\Delta A & \Delta B C_{k 1} \\
\Delta B_{k} C_{1} & \Delta A_{k}
\end{array}\right]=\bar{E}_{1} H \bar{F}_{1}+\bar{E}_{2} H \bar{F}_{2}, \\
\bar{E}_{1}=\left[\begin{array}{c}
E_{1} \\
0
\end{array}\right], \bar{E}_{2}=\left[\begin{array}{c}
0 \\
E_{2}
\end{array}\right], \bar{F}_{1}=\left[\begin{array}{ll}
F_{1} & F_{2} C_{k 1}
\end{array}\right],
\end{gathered}
$$

About the above closed-loop system (4)'s dynamic performance, the quadratic matrix inequality area $D$ considered in this paper is a complex point set on a complex plane which satisfies the following formula ${ }^{[6]}: D=\left\{z \in C: R_{11}+R_{12} z+R_{12}^{T} z^{*}+R_{22} z z^{*}<0\right\}$.

Where $R_{11}, R_{12}, R_{22} \in R^{d \times d}, R_{11}$ and $R_{12}$ are symmetric matrixes, $d$ is called the rank of area $D$. Visibly, $D$ is a convex region which is symmetrical about the real axis, and the left half complex plane and any disc area on the left half complex plane are both particular cases of this kind of convex regions.

1) Definition 1: If the eigenvalues of $A$ are located in area $D$ in the complex plane, that is $\sigma(A) \subset D$, it is called $A$ is stable about $D$.

2) Definition 2: Consider model (1) and all the admissible uncertainties (3). If there exists a feedback tracking system (2) so as to:

a) In the absence of external disturbances, the tracking error of system (4) is asymptotically stable;

b) At zero initial conditions, system (4) has $H_{\infty}-\gamma$ performance;

c) System (4) is stable.

then system (2) is called a non-fragile $H_{\infty}$ tracking control system of model (1) based on output feedback.

The task of this paper is to design a non-fragile tracking control system as form (2) for model (1).

Lemma 1: ${ }^{[6]}$ Matrix $A \in C^{n \times n}$ is $D$ Stable if and only if there exists a symmetric positive definite matrix $P>0$ so as to:

$D(P, A)=R_{11} \otimes P+R_{12} \otimes(P A)+R_{12}^{T} \otimes\left(A^{T} P\right)+R_{22} \otimes\left(A^{T} P A\right)<0$

Lemma 2: Let $\Phi$ be a given symmetric matrix, $H$ and $G$ are the matrixes with appropriate dimensions, then for all the matrix function $F(t)$ which satisfies $F T(t) F(t) \leq I$, the necessary and sufficient conditions for the inequality $\Phi+H F(t) G+G^{T} F^{T}(t) H^{T}<0$

existence of a scalar $\quad \varepsilon>0$ which makes $\Phi+\varepsilon H H^{T}+\varepsilon^{-1} G^{T} G<0$ tenable.

\section{MAIN RESULTS}

Theorem 1: Closed-loop system (4) is quadratic $D$ stable, if and only if there exists a symmetric matrix $X>0$ and two scalars $\varepsilon_{1}, \varepsilon_{2}>0$ which make

$$
\left[\begin{array}{cccc}
\Theta_{11} & \Theta_{12} & I_{d} \otimes X \bar{F}_{1}^{T} & I_{d} \otimes X \bar{F}_{2}^{T} \\
* & \Theta_{22} & 0 & 0 \\
* & * & -\varepsilon_{1} I & 0 \\
* & * & * & -\varepsilon_{2} I
\end{array}\right]<0
$$

Where

$$
\begin{aligned}
& \Theta_{11}=R_{11} \otimes X+R_{12} \otimes \bar{A} X+R_{12}^{T} \otimes X \bar{A}^{T}+\varepsilon_{1} R_{12} R_{12}^{T} \otimes \bar{E}_{1} \bar{E}_{1}^{T}+\varepsilon_{2} R_{12} R_{12}^{T} \otimes \bar{E}_{2} \bar{E}_{2}^{T}, \\
& \Theta_{12}=L \otimes X \bar{A}^{T}+\varepsilon_{1} R_{12} L \otimes \bar{E}_{1} \bar{E}_{1}^{T}+\varepsilon_{2} R_{12} L \otimes \bar{E}_{2} \bar{E}_{2}^{T},
\end{aligned}
$$
$\Theta_{22}=-I_{d} \otimes X+\varepsilon_{1} L^{T} L \otimes \bar{E}_{1} \bar{E}_{1}^{T}+\varepsilon_{2} L^{T} L \otimes \bar{E}_{2} \bar{E}_{2}^{T}$.

Proof: Take $R_{22}=L L^{T}$, let $P=X^{-1}$, then make a congruent transformation for formula (6) with $X$, by substitution of the system matrix $\widehat{A}$, and use Schur 
complement lemma ${ }^{[7]}$ and Lemma 2 to complete the proof, where $\bar{A}=\bar{A}+\Delta \bar{A}$.

Theorem 2: Suppose $R_{11}, R_{12}, R_{22}>0$, for closed-loop system (4), area $D$, and real number $\gamma>0$. If there exists a positive definite symmetric matrix $X>0$ and two scalars $\varepsilon_{1}, \varepsilon_{2}>0$ which make

$$
\left[\begin{array}{cccc}
\Gamma_{11} & \Gamma_{12} & I_{d} \otimes X \bar{F}_{1}^{T} & I_{d} \otimes X \bar{F}_{2}^{T} \\
* & \Gamma_{22} & 0 & 0 \\
* & * & -\varepsilon_{1} I & 0 \\
* & * & * & -\varepsilon_{2} I
\end{array}\right]<0
$$

then system (4) meets performances a), b), and c) where

$$
\begin{array}{r}
\Gamma_{11}=\Theta_{11}+\gamma^{-1} R_{12} \otimes\left(X \bar{C}^{T} \bar{C} X\right)+\gamma^{-1} R_{12} \otimes\left(\bar{D} \bar{D}^{T}\right), \\
\Gamma_{12}=\Theta_{12}, \Gamma_{22}=\Theta_{22} .
\end{array}
$$

Proof: Firstly, the establishment of Formula (8) must have Formula (7) established, so the closed-loop system (4) is $D$ stable. Secondly, from the inverse process of proof for Theorem 1, can we know that formula (8) is equivalent to $D(P, \widehat{A})+\gamma^{-1} R_{12} \otimes\left(X \bar{C}^{T} \bar{C} X\right)+\gamma^{-1} R_{12} \otimes\left(\bar{D} \bar{D}^{T}\right)<0$.

Since $R_{11}, R_{12}, R_{22}>0$ and $P, X>0$, we can obtain $\widehat{A}^{T} P+P \widehat{A}+\gamma^{-1} P \bar{D} \bar{D}^{T} P+\gamma^{-1} \bar{C}^{T} \bar{C}<0$.

Then the conclusion would be achieved by the bounded real theorem ${ }^{[8]}$ : System (4) is asymptotically stable with $H_{\infty}$ performance. QED.

The following derivation is based on the design method of output feedback filter system (2). Formula (8) is non-linear for matrix variable $X$, so use Schur complement to express formula (8) as its equivalent

$$
\left[\begin{array}{ll}
\Psi_{1} & \Psi_{2} \\
* & \Psi_{3}
\end{array}\right]<0
$$

Where

$$
\begin{gathered}
\Psi_{1}=\left[\begin{array}{cccc}
\Psi_{11} & L \otimes X \bar{A}^{T} & R_{12}^{\frac{1}{2}} \otimes\left(X \bar{C}^{T}\right) & R_{12}^{\frac{1}{2}} \otimes \bar{D} \\
* & -I_{d} \otimes X & 0 & 0 \\
* & * & -\gamma I & 0 \\
* & * & * & -\gamma I
\end{array}\right], \\
\Psi_{11}=R_{11} \otimes X+R_{12} \otimes \bar{A} X+R_{12}^{T} \otimes X \bar{A}^{T}, \\
0
\end{gathered}
$$

Thus it can be concluded as follow.

Theorem 3: Suppose a quadratic matrix inequalities region $D$ and a constant $\gamma>0$, for closed-loop system (4), considering the perturbation form as formula (3), if there exist constants $\varepsilon_{i}>0(i=1,2)$, symmetric positive definite matrixes $S, T \in R^{n \times n} \quad, \quad$ and matrixes $U, V \in R^{n \times n} \quad, \quad Y \in R^{n \times n} \quad, \quad Z \in R^{n \times q} \quad, \quad N_{1} \in R^{n \times p} \quad$ and $N_{2} \in R^{n \times m}$, which make

$$
\left[\begin{array}{ll}
\Upsilon_{1} & \Upsilon_{2} \\
* & \Upsilon_{3}
\end{array}\right]<0
$$

then system (4) satisfies performance indices a), b), and c) where

$$
\begin{aligned}
& \Upsilon_{1}=\left[\begin{array}{cccc}
\Upsilon_{11} & \Upsilon_{12} & \Upsilon_{13} & \Upsilon_{14} \\
* & \Upsilon_{22} & 0 & 0 \\
* & * & -\gamma I & 0 \\
* & * & * & -\gamma I
\end{array}\right] \\
& \Upsilon_{11}=R_{11} \otimes \Phi_{22}+R_{12} \otimes \Phi_{12}+R_{12}^{T} \otimes \Phi_{12}^{T}, \Upsilon_{12}=K \otimes \Phi_{12}, \\
& \Upsilon_{22}=-I_{d} \otimes \Phi_{22} \text {, } \\
& \Upsilon_{13}=R_{12}^{\frac{1}{2}} \otimes \Phi_{13}, \Upsilon_{14}=R_{12}^{\frac{1}{2}} \otimes \Phi_{14}, \\
& \Upsilon_{2}=\left[\begin{array}{cccc}
I_{d} \otimes \Phi_{15} & I_{d} \otimes \Phi_{16} & R_{12} \otimes \Phi_{17} & R_{12} \otimes \Phi_{18} \\
0 & 0 & L^{T} \otimes \Phi_{17} & L^{T} \otimes \Phi_{18} \\
0 & 0 & 0 & 0 \\
0 & 0 & 0 & 0
\end{array}\right], \\
& \Upsilon_{3}=\operatorname{diag}\left\{-\varepsilon_{1} I,-\varepsilon_{2} I,-\varepsilon_{1}^{-1} I,-\varepsilon_{2}^{-1} I\right\}, \\
& \Phi_{12}=\left[\begin{array}{cc}
S A+Z C_{1} & Y \\
A & A T+B N_{1}^{T}
\end{array}\right], \Phi_{22}=\left[\begin{array}{cc}
S & I \\
I & T
\end{array}\right], \\
& \Phi_{13}=\left[\begin{array}{c}
C_{2}^{T} \\
T C_{2}^{T}-U C_{k 2}^{T}
\end{array}\right], \Phi_{14}=\left[\begin{array}{c}
S D \\
D
\end{array}\right], \\
& \Phi_{15}=\left[\begin{array}{c}
F_{1}^{T} \\
T F_{1}^{T}+U C_{k 1}^{T} F_{2}^{T}
\end{array}\right], \Phi_{16}=\left[\begin{array}{c}
C_{1}^{T} F_{2}^{T} \\
T C_{1}^{T} F_{2}^{T}+U F_{1}^{T}
\end{array}\right], \\
& \Phi_{17}=\left[\begin{array}{c}
S E_{1} \\
E_{1}
\end{array}\right], \Phi_{18}=\left[\begin{array}{c}
V E_{2} \\
0
\end{array}\right] . \\
& \text { Proof: Disassemble } X \text { and } X^{-1} \text { to } X=\left[\begin{array}{cc}
T & U \\
U^{T} & *
\end{array}\right] \text {, } \\
& X^{-1}=\left[\begin{array}{cc}
S & V \\
V^{T} & *
\end{array}\right] \text {, where } S, T \in R^{n \times n}, U, V \in R^{n \times k}, S, T \text { are }
\end{aligned}
$$




$$
V U^{T}=I-S T
$$

Make a congruent transformation for inequation (9) with $\operatorname{diag}\left\{X_{1}, X_{1}, I, I, I, I, I, I\right\}$, integrate formula (11) and let

$$
\left\{\begin{array}{l}
W=V A_{k}, Z=V B_{k} \\
N_{1}=U C_{k 1}^{T}, N_{2}=U C_{k 2}^{T} \\
Y=S A T+Z C_{1} T+S B N_{1}^{T}+W U^{T}
\end{array}\right.
$$

Then we can obtain formula (10).

So formula (10) is a linear matrix inequality on matrix variable $S, T, Y, Z, N_{1}, N_{2}, U$ and $V$. By integrating (12) with the constraint of formula (11), we can calculate the gain matrixes $A_{k}, B_{k}, C_{k 1}$, and $C_{k 2}$ of output feedback filter system (2) which make closed-loop system (4) meet performances $a), b)$, and c).

\section{EXAMPLE AND SIMULATION}

Consider a certain type unmanned airship in the height of $500 \mathrm{~m}$, speed $12 \mathrm{~m} / \mathrm{s}$, longitudinal motion parameters:

$$
\begin{array}{r}
A=\left[\begin{array}{ccc}
-2.5285 & -0.1051 & 0.4875 \\
0.5063 & -2.1027 & -0.0096 \\
-0.2583 & 0.3018 & -2.7586
\end{array}\right], B=\left[\begin{array}{c}
-2.0863 \\
1.0683 \\
-5.5902
\end{array}\right], \\
D=\left[\begin{array}{c}
-0.5285 \\
0 \\
-1.0583
\end{array}\right], C_{1}=\left[\begin{array}{lll}
-0.2 & 0.3 & 0.2
\end{array}\right], \\
C_{2}=\left[\begin{array}{lll}
0.3 & -0.2 & 0.2
\end{array}\right],
\end{array}
$$

Uncertain parameters: $\quad E_{1}=\left[\begin{array}{lll}0.6 & 0.8 & 0.7\end{array}\right]^{T}$, $E_{2}=\left[\begin{array}{lll}0.04 & 0.06 & 0.08\end{array}\right]^{T}, F_{1}=\left[\begin{array}{lll}0.5 & 0.5 & 0.5\end{array}\right], F_{2}=0.8$.

It is required that the pole of closed-loop system (4) be placed in circle area $D(-4,3.6)$ of the left half complex plane, interference suppression indicators $\gamma=0.5$. According to Theorem 2, appropriately selected the values which make $\varepsilon_{1}, \varepsilon_{2}>0$, with the precondition that formula (10) has a feasible solution, the gain matrixes of output feedback filter system (2) which make closed-loop system (4) satisfy performances a), b), and c) are

$$
\begin{gathered}
A_{k}=\left[\begin{array}{ccc}
-1.5904 & -3.1040 & 0.6173 \\
0.8011 & -2.6193 & 1.2169 \\
-0.2587 & 0.0127 & -2.9714
\end{array}\right], B_{k}=\left[\begin{array}{c}
24.8954 \\
16.7084 \\
-6.0162
\end{array}\right], \\
C_{k 1}=\left[\begin{array}{lll}
-0.0023 & 0.0202 & -0.3613
\end{array}\right], \\
C_{k 2}=\left[\begin{array}{lll}
0.0302 & -0.1375 & -1.1824
\end{array}\right] .
\end{gathered}
$$

Take $H(t)=\sin (t), t$ is an arbitrarily real number, which makes $H^{T}(t) H(t) \leq I$, then the pole group distribution of closed-loop system (4) at each time point is obtained as shown in Fig. 1. Given the system's initial state $x=\left[\begin{array}{lll}0.2 & -0.2 & 0.3\end{array}\right]$, and in the absence of external disturbances, the status response curve of closed-loop system (4) can be obtained as shown in Fig. 2.

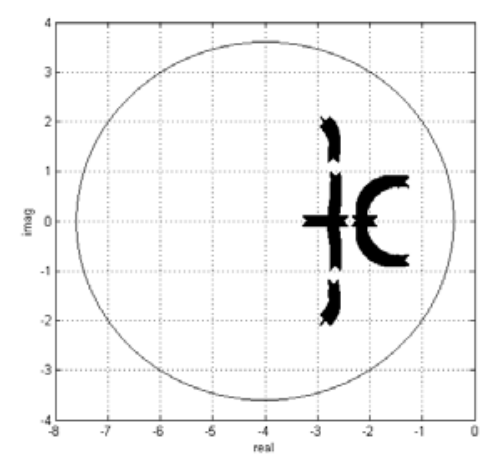

Figure 1. Pole distribution of closed-loop system.

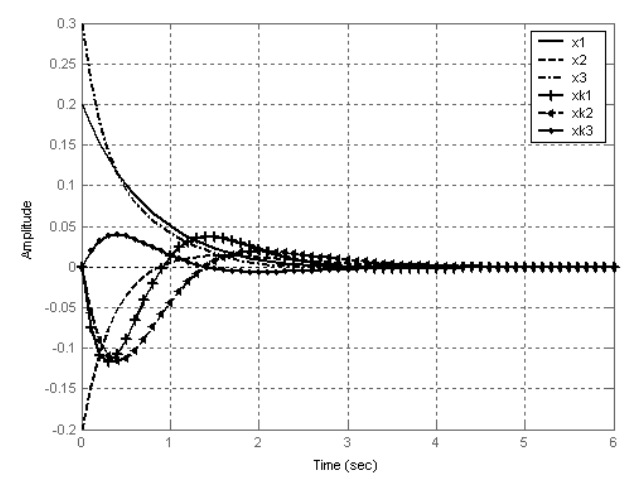

Figure 2. Status response curve.

From Fig. 1 can we know that the poles of closed-loop system are located within the specified area. Fig. 2 shows that system is asymptotically stable. Thus the design goal is achieved.

\section{CONCLUSION}

Considering the case of uncertainties in both controlled system and feedback filtering system, the closed-loop system designed for controlling UAV models is asymptotically stable, filtering error output for interference suppression performance is less than a given upper limit, and closed-loop system pole were placed in the designated area on the complex plane. Solvability condition of output feedback filter system can be obtained in the form of LMI, and the design method of non-fragile filter system was advanced. A longitudinal motion simulation was carried out for a certain type of unmanned airship using this method, and the simulation results show the effectiveness of the method.

\section{ACKNOWLEDGEMENT}

This research was financially supported by the National Science Foundation under grant \#61374188. 


\section{REFERENCES}

[1] Zhou Wuneng, Su Hongye, Chu Jian. Robust H-infinity Output Feedback Control with Variance and Pole Constraints for Time-varying Uncertain systems [J]. Control Theory \& Applications, 2007, 24(1): 103-108.

[2] Yang Xiaoguang, Zhang Qingling, Li Li. Static Output Feedback Control for Continuous Uncertain Fuzzy Systems [J]. Control and Decision, 2008, 23(5): 585-588.

[3] Yang Guanghong, Wang Jianliang. Robust non-fragile kalman filtering for uncertain linear systems with estimation gain uncertainty [J]. IEEE Transaction on Automatic Control 2001, 46(2): 343-348.
[4] Yang Guanghong, Che Weiwei. Non-fragile H-infinity filter design with additive gain variations [C] Proceedings of 45th IEEE Conference on Decision and Control San Diego. 2006: 4775-4780.

[5] Wang Wu, Guo Xianggui, Yang Fuwen. Non-fragile H-infinity filtering for linear system[J], Control and Decision, 2008, 23(5): 503-506.

[6] Valter J, Leite S, Pedro L. An improved LMI condition for robust D-stability of uncertain polytopic systems [J]. IEEE Trans on Automatic Control, 2003, 48(3): 500-504.

[7] nformation on https://en.wikipedia.org/wiki/Schur_complement.

[8] Zheng Min, Xiao Wei, Li Yang. Analysis of Linear Systems with Time Delay in Window Ho Frequency Domain [J]. Control Engineering of China, 2012, 19(5): 896-899. 